\title{
Solid-phase Diffusion Mechanism for GaAs Nanowire Growth
}

\author{
Magnus W. Larsson*, Ann I. Persson ${ }^{* *}$, L. Reine Wallenberg ${ }^{*}$, Lars Samuelson ${ }^{* *}$ \\ * nCHREM/Materials Chemistry, Lund University, Box 124, S-221 00 Lund, Sweden \\ ** Solid State Physics, Lund University, Box 118, S-221 00 Lund, Sweden
}

In-situ heating of nanowires has been performed in order to understand the process of catalysed nanowire growth. The proposed growth process has been described as the vapour-liquid-solid (VLS) mechanism, where the epitaxial crystal growth is catalysed by a molten gold droplet in a vapour of the reagents onto a solid substrate surface. However, in-situ heating of nanowires shows a solid state gold/gallium particle at the growth temperature indicating that nanowires can be grown catalyzed by a solid particle instead of a liquid droplet.[1]

GaAs nanowires were grown in a chemical beam epitaxy (CBE) system at $540^{\circ} \mathrm{C}$ with gold nano particles as growth catalysts [2]. After growth, the wires were removed from the substrate and deposited onto a carbon lacy grid. The wires were heated in a heating stage and images were acquired at different temperatures with a transmission electron microscope (TEM) and analyzed with energy dispersive spectrometry (EDS).

The nanowire growth takes place at the surface between the gold seed particle and the crystalline surface forming a wire with the same diameter as the gold particle. At the end of the growth process the temperature is lowered and the gallium supply is terminated while keeping the arsenic pressure constant. The last part of the growth will then form a characteristic 'neck' right below the gold particle (Fig. 1a). To investigate the origin of the neck the wires were re-heated to probe whether we could re-alloy the material of the neck with the metal particle. In the first experiment nanowires were slowly heated up to $470^{\circ} \mathrm{C}$ over a four-hour period while continuously observing them in the TEM. The wires were then kept above $400^{\circ} \mathrm{C}$ for another four hours and then finally cooled down to room temperature (Fig. 1a-c). Several very clear observations were made. There was a noticeable alloying between the gold particle and the wire already at temperatures around $350^{\circ} \mathrm{C}$. We also observed that keeping the wires at temperatures above $400^{\circ} \mathrm{C}$ lead to a continuing decomposition of the nanowires (Fig. 1b-c). No change in appearance, i.e. no new neck formation was observed on cooling down the wires to room temperature, indicating that the arsenic was not stored in the gold particle and the reversed reaction could evidently not take place.

This result was also confirmed by EDS line scans (Fig. 2a-b) comparing nanowires before and after the heating experiments. The measurements showed both gallium and arsenic to be absent in the seed particle before heating, whereas gallium was present in the seed particles after heating. From these results we conclude that the neck in the nanowires was created after the gallium source had been switched off allowing the gallium inside the gold particle to react with the arsenic still present in the growth chamber.

A second heating experiment was carried out, where nanowires were heated to above $600^{\circ} \mathrm{C}$ in the TEM. Images and diffraction patterns acquired at $540^{\circ} \mathrm{C}$, which is equivalent to the growth temperature, showed crystallinity of the seed particle (Fig. 3). This was also seen at $600^{\circ} \mathrm{C}$, giving strong evidence for the seed particle being in solid state during growth.

\section{References}

[1] A. I. Persson, M. W. Larsson et. al. Nature Materials 3 (2004) 677-681

[2] B. J. Ohlsson et. al. , Appl. Phys. Lett., 79, 3335 (2001) 


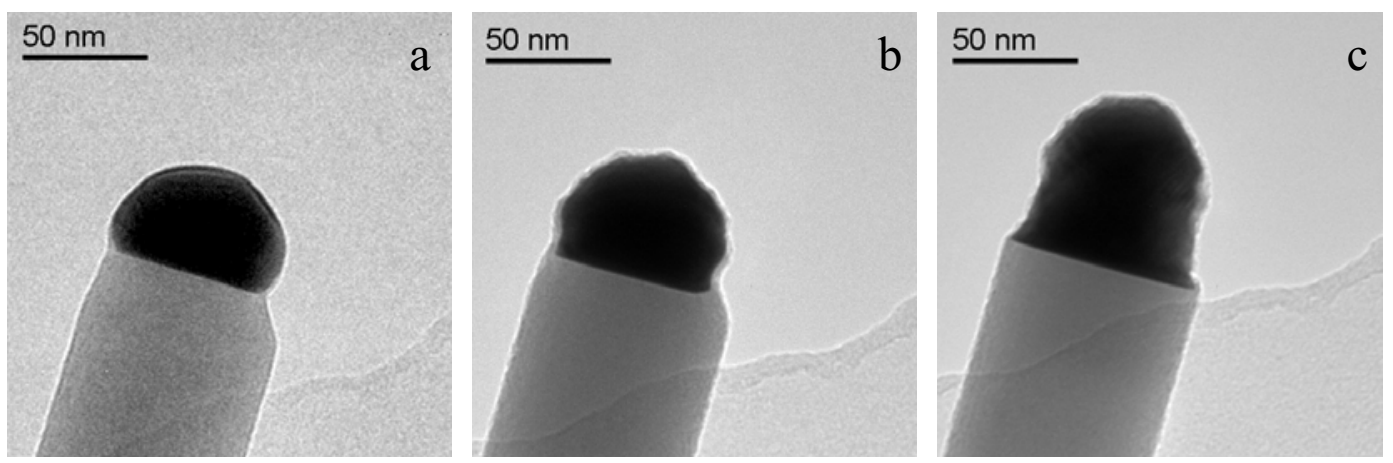

FIG. 1. In-Situ heating of a GaAs nanowire. a, At room temperature, the neck is clearly visible. b, At $440^{\circ} \mathrm{C}$, the gold particle has started the decomposition of the nanowire. c, At $440^{\circ} \mathrm{C}$ after five hours of heating, the gold particle has migrated beyond the neck.

a

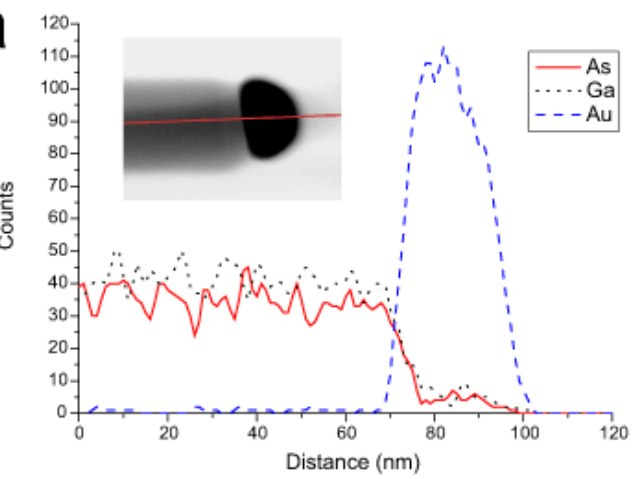

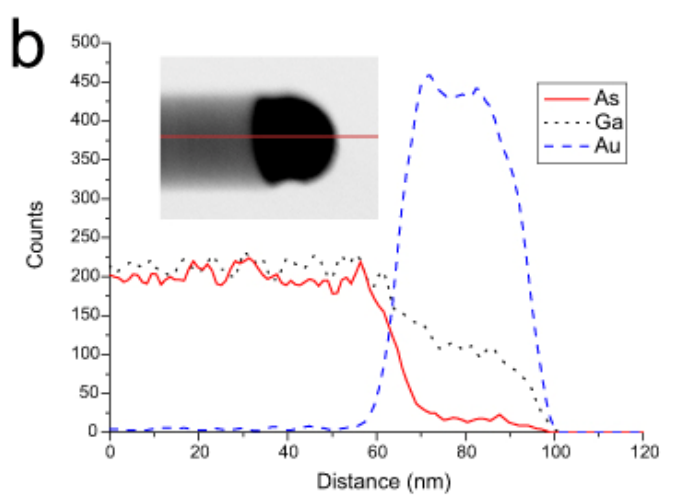

FIG. 2a, EDS line scan along a non-heated GaAs nanowire. No gallium or arsenic is present in the gold particle resulting from transport of gallium atoms in the alloyed seed particle into the neckregion during cooling-down under arsenic pressure at the end of growth. $2 b$, EDS line scan along a heated GaAs nanowire. The gold particle contains gallium but no arsenic, resulting from the reformation of the gold-gallium alloy due to transport of gold into the neck-region.

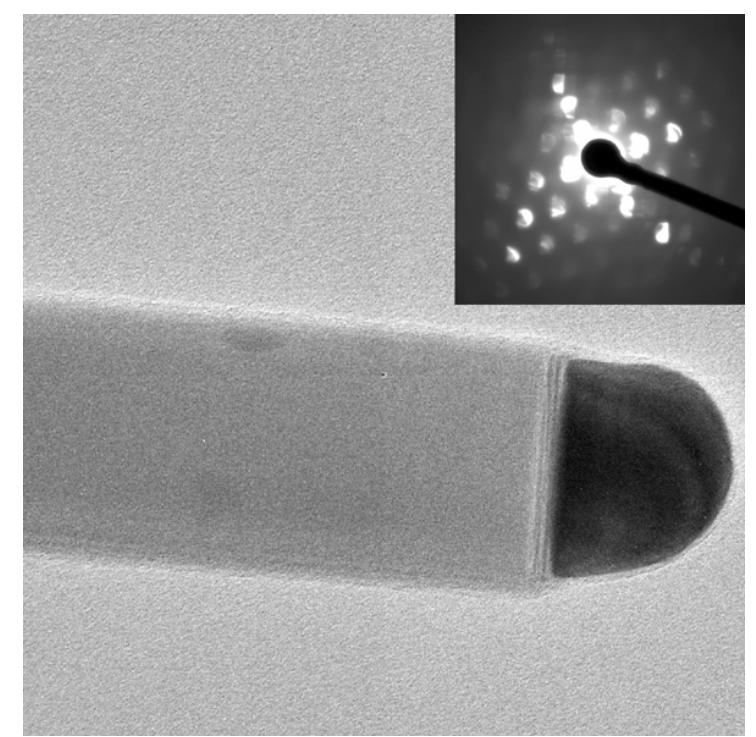

FIG. 3. GaAs nanowire at $540^{\circ} \mathrm{C}$ (growth temperature). Diffraction pattern inserted showing reflections from the gold/gallium particle as well as the GaAs nanowire. 